\title{
Perioperative management of obstructive sleep apnea: Ready for prime time?
}

\section{ABSTRACT}

Obstructive sleep apnea (OSA) is associated with increased risks of cardiovascular disease and stroke and with elevated rates of postoperative complications (including cardiac ischemia and respiratory failure) in surgical patients. Additionally, the prevalence of OSA is higher in surgical patients than in the general population. Screening for OSA prior to surgery is recommended to identify patients at risk for postoperative complications. The presence of moderate or severe OSA calls for modified strategies of perioperative anesthesia, pain management, and postoperative monitoring to reduce the chance of OSA-associated complications.

\section{KEY POINTS}

OSA is more common than asthma in adults, affecting $4 \%$ and $2 \%$ of middle-aged men and women, respectively.

OSA is associated with serious health consequences, including increased risks for accidents, stroke, hypertension, coronary artery disease, atrial fibrillation, and postoperative complications.

Screening tools consisting of only a few questions are available to quickly and effectively identify risk for OSA prior to surgery.

For surgical patients deemed to be at high risk for OSA, and for whom surgery cannot be delayed for diagnostic tests and OSA treatment, the best course is to proceed with surgery but assume the patient has moderate to severe OSA.

Use of regional anesthesia, close attention to airway management, vigilant postoperative monitoring of pulse oximetry, and minimal use of opioids are recommended for patients with OSA.
0 bstructive sleep apnea (OSA) is characterized by repeated complete or partial collapse of the pharyngeal airway during sleep, causing cessation of airflow (apnea) or shallow breathing (hypopnea). Persons with OSA may have repeated arousals from sleep (to reestablish breathing) with each episode of apnea or hypopnea. The resulting sleep disruption often leads to daytime somnolence and compromised neurocognitive function.

This pattern of sleep arousal, coupled with intermittent hypoxemia, is associated with serious adverse cardiovascular outcomes, including stroke. Among surgical patients, OSA is associated with postoperative complications and the need for increased medical intervention. This review discusses why OSA is important in the perioperative setting, preoperative screening for OSA risk, and perioperative management of patients with likely or confirmed OSA.

\section{OSA AT A GLANCE}

\section{Prevalence in the general population}

Four percent of middle-aged men and $2 \%$ of middleaged women meet minimal diagnostic criteria for OSA, according to a landmark cohort study from the 1990s. ${ }^{1}$ This makes OSA more common than asthma among adults. Risk increases with age, as $24 \%$ of persons older than 65 years have OSA and up to 50\% of nursing home residents have clinically significant OSA. ${ }^{2}$

\section{Prevalence in the surgical population}

The prevalence of OSA in the surgical population is higher than that in the general population, and it can vary widely according to the underlying medical condition. A study of 433 patients undergoing general surgery reported a $3.2 \%$ prevalence of OSA, ${ }^{3}$ but this study excluded patients undergoing cardiac surgery, in whom the risk of OSA is higher. In contrast, the prevalence of OSA among obese bariatric surgery patients has been reported at greater than 70\%. Notably, the patients in the general surgery study ${ }^{3}$ who appeared to be at risk for OSA based on screening questions were invited to participate in a sleep study, whereas all patients in the bariatric surgery study ${ }^{4}$ were evaluated through sleep studies. It is likely that the prevalence of OSA among the general surgery study patients would 
have been higher if all patients had been evaluated with polysomnography.

\section{Pathophysiology}

OSA can occur when any part of the upper airway does not function normally. Upper airway patency is determined by muscle activity, craniofacial and soft tissue structure, and sleep state. During sleep, upper airway muscles are relaxed, which reduces airway patency. Sleep is associated with pharyngeal narrowing and substantially increased inspiratory resistance even among persons without sleep apnea. A person who is awake can compensate for abnormal pharyngeal function through increased muscle activity. During sleep this muscle compensation fails, resulting in partial collapse and subsequent snoring, and sometimes prolonged obstructive hypoventilation. Complete closure results in apnea.

\section{WHY OSA MATTERS}

\section{Health consequences of OSA}

OSA is associated with serious health consequences, such as increased risk of motor vehicle accidents, stroke, and a number of cardiovascular conditions-hypertension, coronary artery disease, and atrial fibrillation.

Accidents. The daytime hypersomnolence resulting from OSA contributes to reduced vigilance and is likely responsible for an increased incidence of motor vehicle accidents. One study found that among a sample of men and women with unrecognized OSA undergoing polysomnography studies, the likelihood of motor vehicle accidents during the prior 5 years was significantly correlated with the subjects' apnea-hypopnea index (AHI) score, which reflects the number of apnea or hypopnea episodes per hour of sleep. ${ }^{5}$ Other studies have demonstrated similar associations.

Stroke. Numerous observational studies have demonstrated an elevated prevalence of OSA among patients with stroke as compared with the general population, but these studies did not adjust for other cerebrovascular risk factors. A recent observational cohort study aimed to address this evidence gap by using proportional hazards analysis to determine the independent effect of OSA on the incidence of stroke or death from any cause among persons with no history of stroke or myocardial infarction. ${ }^{6}$ Study participants were 1,022 consecutive patients who underwent polysomnography for evaluation of sleepdisordered breathing. OSA was identified in $68 \%$ of patients. During the 3.4-year follow-up period, 22 strokes and 50 deaths occurred among the 697 patients with OSA compared with 2 strokes and 14 deaths among the 325 patients without OSA. The probability of survival was significantly lower for patients with OSA compared with their counterparts without OSA $(P<.003)$. After adjustment for other risk factors, OSA was significantly associated with stroke or death (hazard ratio $=1.97 ; 95 \%$ CI, 1.12-3.48). ${ }^{6}$

Hypertension. Four large studies involving a total of 10,708 patients evaluated for sleep-disordered breathing have established an association between OSA and hypertension risk. ${ }^{7-10}$ In each study, the risk of hypertension rose linearly with AHI scores. Clinically significant OSA, defined as an AHI score greater than 15 , roughly doubled the risk of hypertension compared with the absence of apnea/hypopnea episodes, with odds ratios ranging from 1.37 to 2.89 across the four studies. ${ }^{7-10}$ Each apnea event per hour of sleep was estimated to increase the odds of developing hypertension by approximately $1 \% .{ }^{8}$ Notably, the effects of OSA on blood pressure are most pronounced in patients younger than age $50 . ?$

Coronary artery disease. The Sleep Heart Health Study evaluated the association between sleep-disordered breathing and cardiovascular disease in 6,424 communitydwelling adults undergoing home polysomnography. ${ }^{11}$ The population's median AHI score was 4.4. At least one cardiovascular event was reported by $16 \%$ of participants. Sleep-disordered breathing was associated with self-reported heart failure, stroke, and, more modestly, coronary artery disease. A linear relationship was noted between $\mathrm{AHI}$ and cardiovascular risk.

Snoring, which is often an indicator for OSA, has also been associated with cardiovascular risk. The Nurses' Health Study evaluated 71,000 women who completed medical questionnaires that included questions about snoring. Over 8 years of follow-up, the relative risks for cardiovascular disease were 1.46 among occasional snorers (95\% CI, 1.23-1.74) and 2.02 among regular snorers (95\% CI, 1.62-2.53) in comparison with nonsnorers. Snoring, even without a diagnosis of OSA, emerged as an independent risk factor for cardiovascular disease. ${ }^{12}$

Atrial fibrillation. OSA has been identified as a predictor of new-onset atrial fibrillation in a retrospective cohort study (hazard ratio $=2.18 ; 95 \%$ CI, 1.34-3.54) ${ }^{13}$ In a prospective study, patients with atrial fibrillation but normal left ventricular function were found to have significantly higher AHI scores than matched normal controls. ${ }^{14}$ After adjustment for relevant covariates, the odds ratio for an association between atrial fibrillation and significant sleep-disordered breathing (AHI score > 15) was 3.04 (95\% CI, 1.24-7.46)..$^{14}$ In another prospective trial, patients with atrial fibrillation and OSA who underwent cardioversion were at increased risk for a recurrence of atrial fibrillation if OSA was untreated $(82 \%$ for untreated vs $42 \%$ for treated OSA; $P=.013) .{ }^{15}$

\section{An association with postoperative complications}

OSA also has been shown to increase postoperative complication rates, increase the need for intensive care intervention, and prolong hospital stays. 
TABLE 1

Factors to keep in mind in the evaluation for obstructive sleep apnea (OSA)

Factors that reduce upper airway size or predispose to upper airway collapse

Obesity

Male gender

Menopausal status

Hard-tissue craniofacial abnormalities (retrognathia, micrognathia, brachycephaly)

Soft-tissue craniofacial abnormalities (large uvula, enlarged tonsils, macroglossia, long soft palate)

Alcohol or sedative use (aggravates underlying OSA)

Symptoms and complaints that may be suggestive of OSA

Snoring

Personality change

Sleepiness

Morning confusion

Physically restless sleep

Night sweats

Intellectual impairment

Morning dry mouth or sore throat

Impotence

Morning headaches

Representative evidence. One of the first studies to characterize the postoperative risks of OSA was conducted by Mayo Clinic researchers who retrospectively reviewed 4 years of data for 101 patients with OSA who had had hip or knee replacement surgery within 3 years before $(n=36)$ or any time after $(n=65)$ their OSA diagnosis. ${ }^{16}$ Outcomes were compared with those of 101 matched controls without OSA who underwent the same operations. Only half the patients with diagnosed OSA prior to their operation used continuous positive airway pressure (CPAP) therapy at home prior to hospitalization. Complications occurred among 39\% of patients with OSA and among $18 \%$ of control patients $(P=.001)$. Serious complications requiring intensive care unit transfer for cardiac ischemia or respiratory failure occurred in $24 \%$ of patients with OSA versus only $9 \%$ of controls $(P=.004)$, and hospital stays were longer for patients with OSA compared with controls $(P<.007)$. Most complications occurred during the first day after surgery, but a small number occurred as late as postoperative days 4 and 5 .

In a separate study designed to evaluate OSA screening tools, postoperative complication rates were assessed in 211 patients who underwent polysomnography to determine the presence or absence of OSA prior to elective surgery. ${ }^{17}$ Patients undergoing various elective procedures were included, but none were undergoing cardiac or bariatric procedures. The overall rate of post- operative complications was more than twice as high among patients with OSA compared with those without OSA $(27.4 \%$ vs $12.3 \% ; P=.02)$. The most common complication was oxygen desaturation (ie, level $\leq 90 \%$ ), which occurred among $20.6 \%$ of patients with OSA versus $9.2 \%$ of patients without OSA $(P<.04)$. There were no deaths or serious complications.

Potential causes of complications. In the immediate postoperative period, OSA-associated complications may be attributable to lingering effects of sedatives, which can often lead to respiratory problems. Later in the postoperative course, so-called REM rebound is more likely to be implicated in complications. Patients often experience sleep deprivation in the hospital due to constant interruptions. Once a patient does sleep, the amount of REM sleep increases to compensate for this deprivation. The REM stage is when most apneas and hypopneas occur, so the risk of hypoxemia is greatest in the REM stage. As a result, respiratory and cardiovascular complications such as arrhythmias can increase.

\section{OSA AND THE PREOPERATIVE EVALUATION}

\section{Risk factors for OSA}

The top portion of Table 1 lists factors that reduce upper airway size or predispose to upper airway collapse and thereby increase risk for OSA. Fortunately, anesthesiologists are frequently aware of the craniofacial abnormalities listed in the table because they affect ease of intubation. The inclusion of menopausal status reflects the fact that women tend to catch up with men in their risk for OSA by the time they reach menopause.

Additionally, certain aspects of perioperative management can increase the risk of OSA in the perioperative setting. For example, general anesthesia can mimic the effects of sleep on the airway, reducing muscle tone and potentially leading to pharyngeal collapse. Normal response to hypercapnia is also diminished under general anesthesia and while patients remain sedated postoperatively, which subdues normal protective arousal mechanisms. This does not pose a problem while the patient remains intubated but highlights the need for respiratory monitoring in the extubated patient who is recovering from the residual effects of sedation.

\section{History and physical examination}

What to look for. A number of physical characteristics reveal potential risks for OSA. Obesity and hypertension are well established, as noted above. Large neck circumference ( $\geq 17$ inches in men and $\geq 16$ inches in women) is another characteristic associated with OSA. Examination of the upper airway can reveal obstruction due to tonsil enlargement, nasal obstruction, an elongated uvula, or macroglossia. Since retrognathia 
or micrognathia can produce a narrowed oropharynx, attention to mandible size and position is advised.

Ask about sleep habits. Assessment of OSA risk in the preoperative evaluation need not be lengthy, but patients should be asked about snoring and waking habits, especially frequency of night wakening, to identify possible OSA. Patients generally do not volunteer information about sleep, so it is important to explicitly ask. Responses that suggest OSA include reports of tiredness or sleepiness during the day, or comments by a partner about the patient's snoring. A patient who reports having a dry mouth in the morning may have nasal congestion or obstruction that leads to mouth breathing. Severe sleep disruption can lead to sleep deprivation, causing personality changes, confusion, intellectual impairment, impotence, or morning headaches (Table 1 ).

Preoperative screening tools. Screening tools can assist in identifying relevant questions about sleep. Three such tools for OSA have been validated for use in surgical patients: the Berlin questionnaire, the American Society of Anesthesiologists (ASA) checklist, and the STOP questionnaire. ${ }^{17-20}$ The performance of these tools was evaluated in 177 surgical patients with OSA identified using polysomnography. ${ }^{17}$ Each tool's sensitivity, specificity, and positive and negative predictive values were calculated according to polysomnographybased AHI severity. All three tools demonstrated moderately high sensitivity for detecting OSA. ${ }^{17}$

Use of any of these screening tools improves the likelihood of identifying OSA preoperatively. The quickest and simplest to use is the STOP questionnaire, which was recently modified to include questions about additional risk factors for OSA-body mass index, age, neck circumference, and gender; the modified tool is called the STOP-BANG questionnaire (Table 2).${ }^{20}$ In a validation study, the addition of the "BANG" questions about these risk factors increased the questionnaire's specificity for moderate to severe OSA. ${ }^{20}$ It is important to ask the questions as they are written (Table 2 ) to elicit the most complete response. For example, the question "Do you feel tired, fatigued, or sleepy?" may seem redundant, but all three terms should be included because men often complain of feeling sleepy while women are more likely to report feeling tired or fatigued.

Identifying levels of OSA severity. Physical examination and screening questions may be adequate to identify patients at risk for OSA prior to surgery. Mild OSA (AHI score of 5-15) can generally be managed after surgery, at the patient's leisure. In contrast, moderate OSA (AHI score of 15-30) and severe OSA (AHI score > 30) can affect perioperative management (see next section). If moderate to severe OSA is suspected, and if there is enough time before surgery to consult a sleep lab, polysomnography can provide a more complete diagnosis.

\begin{tabular}{|c|c|c|}
\hline \multicolumn{3}{|l|}{ STOP } \\
\hline S (snore) & $\begin{array}{l}\text { Do you snore loudly (louder } \\
\text { than talking or loud enough to } \\
\text { be heard through closed doors)? }\end{array}$ & Yes/No \\
\hline $\mathrm{T}$ (tired) & $\begin{array}{l}\text { Do you often feel tired, fatigued, } \\
\text { or sleepy during daytime? }\end{array}$ & Yes/No \\
\hline 0 (observed) & $\begin{array}{l}\text { Has anyone observed you stop } \\
\text { breathing during sleep? }\end{array}$ & Yes/No \\
\hline $\begin{array}{l}\mathrm{P} \text { (blood } \\
\text { pressure) }\end{array}$ & $\begin{array}{l}\text { Do you have or are you being } \\
\text { treated for high blood pressure? }\end{array}$ & Yes/No \\
\hline \multicolumn{3}{|l|}{ BANG } \\
\hline $\begin{array}{l}\text { B (body mass } \\
\text { index }[\text { BMI]) }\end{array}$ & $B M I>35 \mathrm{~kg} / \mathrm{m}^{2} ?$ & Yes/No \\
\hline A (age) & Age $>50$ years? & Yes/No \\
\hline $\mathrm{N}$ (neck) & Neck circumference $>40 \mathrm{~cm}$ ? & Yes/No \\
\hline G (gender) & Gender male? & Yes/No \\
\hline
\end{tabular}

Yes to $\geqslant 3$ questions $=$ high risk of obstructive sleep apnea Yes to $<3$ questions $=$ low risk of obstructive sleep apnea

${ }^{*}$ Adapted from Chung et al. ${ }^{20}$

\section{PERIOPERATIVE MANAGEMENT OF OSA}

\section{When in doubt, proceed as if patient has OSA}

Evidence of OSA's association with postoperative complications is emerging, as noted above, but more specific information about risks is needed to develop effective management procedures. For surgical patients who are deemed to be at high risk for OSA, and for whom surgery cannot be delayed for diagnostic tests and OSA treatment, the most prudent course is to proceed with surgery but assume the patient has moderate to severe OSA. Anesthesiologists should be informed when patients are likely to have OSA, as they may choose a different strategy for managing anesthesia during surgery for patients at high risk.

\section{Management recommendations}

The ASA published practice guidelines in 2006 for the perioperative management of patients with OSA. ${ }^{19}$ In view of the paucity of data on the best management strategies, the guidelines were based mostly on expert opinion. Their key recommendations include the following:

- Surgical patients should be screened clinically to determine their OSA risk. Any of the aforementioned screening tools is effective for this purpose.

- For patients with a diagnosis of OSA or who are clinically determined to be at high risk, close attention to airway management is required, extubation should be 
done when the patient is fully awake (to reduce residual effects of anesthesia and sedatives), and regional anesthesia should be used whenever possible.

- Postoperative pain management in patients with confirmed or suspected OSA should minimize the use of opioids and other sedatives. Such patients also should undergo close pulse oximetry monitoring in a step-down setting after surgery and receive postoperative CPAP therapy as soon as possible.

These ASA recommendations are broadly echoed by a 2003 clinical practice review report of the American Academy of Sleep Medicine, which recommends careful attention during the first 24 hours after surgery in patients with presumed OSA and also cautions that patient-controlled analgesia may not be appropriate. ${ }^{21}$

\section{Future research questions}

Even with the insights reviewed above, many questions about perioperative management of OSA remain, including the following:

- Will the early diagnosis and treatment of OSAusually with CPAP - improve perioperative and postoperative outcomes?

- What are the costs associated with observed complications of OSA, and will immediate and continued use of CPAP postoperatively prove cost-effective?

- Where should patients with OSA be monitored postoperatively, and for how long?

- Which pain-control strategies are best for patients with OSA?

\section{DISCUSSION}

Question from the audience: Have studies of OSAassociated postoperative complications stratified results on the basis of AHI score?

Dr. Shafazand: Yes. In most studies, postoperative complications are more likely to occur among patients with AHI scores that indicate moderate to severe OSA. However, although the AHI is used extensively as a measure of OSA severity, it may not be the best measure. The degree and duration of oxygen desaturation are probably more relevant to the physiologic changes that occur than is the actual apnea or hypopnea event. The more severe the hypoxemia, the greater the risk of complications.

Comment from the audience: I want to reiterate the point from earlier in this summit that consultant physicians should avoid recommending a type of anesthetic in a preoperative consult. Despite the recommendations of the 2006 ASA guidelines, ${ }^{19}$ many anesthesiologists prefer to use a minimal opioid technique or a general anesthetic for patients with OSA rather than risk losing the airway during the operation and having to perform an emergent intubation.
Dr. Shafazand: I agree. In my own consultations I never presume to make recommendations about the type of anesthesia to be used. The important thing is to have a discussion with the anesthesiologist about the best way to manage patients with OSA, but not in the intraoperative context because the patient is going to be intubated and the airway will be protected. The discussion is really more about how to manage patients once they are extubated.

Question from the audience: Should patients with OSA undergo surgery in outpatient facilities?

Dr. Shafazand: It depends on the type and duration of the procedure. If it is a quick procedure, which is likely for an outpatient facility, with minimal sedation and a period of respiratory observation to ensure that the patient is fully awake, the outpatient setting is probably acceptable, especially if the patient is using CPAP at home. It also depends on the severity of OSA. For patients with more severe OSA, an outpatient facility is not recommended. Unfortunately, data about OSA complications in outpatient facilities are sparse.

Question from the audience: What is the role of overnight pulse oximetry versus a sleep study?

Dr. Shafazand: That is the Achilles' heel of managing patients with OSA. Sleep labs are overbooked, so it is often not possible to order a sleep study for patients prior to surgery. Some studies have evaluated overnight pulse oximetry, noting the percentage of desaturation or the total time spent at less than $90 \%$ saturation during the night or per hour. This approach is probably adequate for screening for suspected severe OSA, but not all patients with OSA will have desaturations. Overnight pulse oximetry is at best a "poor man's" screening tool-if it is negative, OSA cannot be ruled out.

Question from the audience: What is your opinion of surgical treatments for sleep apnea such as uvulopalatopharyngoplasty (UPPP)?

Dr. Shafazand: For patients with an AHI score below 15 and no comorbidities, some surgical correction may be advisable. For patients with an AHI score above 15, surgery can be recommended in some circumstancesfor example, if there is a clear blockage of the nasal passage. But patients with moderate to severe OSA usually continue to require CPAP therapy after surgery. CPAP is still the recommended treatment for moderate to severe OSA, though surgery might help the patient tolerate CPAP better in certain instances by lowering the pressure requirements.

Question from the audience: A minimal number of hospitals actually screen patients for OSA and treat them differently. Do you know why the Joint Commis- 
sion dropped a proposed safety goal to screen patients for OSA upon admission and treat based on the results?

Dr. Shafazand: I think the biggest problem is that results from the literature are so variable in terms of risks that it's difficult to draw conclusions. Patients with desaturation are given oxygen to address the immediate problem, but there is no focus on complications. Depending on the study, there are true complications that affect patient safety but also add to the costs of care. Until there are more definitive results in the literature, there is not enough evidence to make and enforce recommendations.

\section{DISCLOSURES}

Dr. Shafazand has indicated that she has no financial relationships with commercial interests that have a direct bearing on the subject matter of this article.

This article was developed from an audio transcript of Dr. Shafazand's lecture at the 4th Annual Perioperative Medicine Summit. The transcript was edited by the Cleveland Clinic Journal of Medicine staff for clarity and conciseness, and was then reviewed, revised, and approved by Dr. Shafazand.

\section{REFERENCES}

1. Young T, Palta M, Dempsey J, Skatrud J, Weber S, Badr S. The occurrence of sleep-disordered breathing among middle-aged adults. N Engl J Med 1993; 328:1230-1235.

2. Young T, Peppard PE, Gottlieb DJ. Epidemiology of obstructive sleep apnea: a population health perspective. Am J Respir Crit Care Med 2002; 165:1217-1239.

3. Fidan H, Fidan F, Unlu M, et al. Prevalence of sleep apnoea in patients undergoing operation. Sleep Breath 2006; 10:161-165.

4. Lopez PP, Stefan B, Schulman CI, Byers PM. Prevalence of sleep apnea in morbidly obese patients who presented for weight loss surgery evaluation: more evidence for routine screening for obstructive sleep apnea before weight loss surgery. Am Surg 2008; 74:834-838.

5. Young T, Blustein J, Finn L, Palta M. Sleep-disordered breathing and motor vehicle accidents in a population-based sample of employed adults. Sleep 1997; 20:608-613.

6. Yaggi HK, Concato J, Kernana WN, et al. Obstructive sleep apnea as a risk factor for stroke and death. N Engl J Med 2005; 353:2034-2041.

7. Grote L, Ploch T, Heitmann J, et al. Sleep-related breathing disorder is an independent risk factor for systemic hypertension. Am J Respir Crit Care Med 1999; 160:1875-1882.

8. Lavie P, Herer P, Hoffstein V. Obstructive sleep apnoea syndrome as a risk factor for hypertension: population study. BMJ 2000; 320:479-482.

9. Nieto FJ, Young TB, Lind BK, et al. Association of sleep-disordered breathing, sleep apnea, and hypertension in a large community-based study. Sleep Heart Health Study. JAMA 2000; 283:1829-1836.

10. Peppard PE, Young T, Palta M, Skatrud J. Prospective study of the association between sleep-disordered breathing and hypertension. N Engl J Med 2000; 342:1378-1384.

11. Shahar E, Whitney CW, Redline S, et al. Sleep-disordered breathing and cardiovascular disease: cross-sectional results of the Sleep Heart Health Study. Am J Respir Crit Care Med 2001; 163:19-25.

12. Hu FB, Willett WC, Manson JE, et al. Snoring and risk of cardiovascular disease in women. J Am Coll Cardiol 2000; 35:308-313.

13. Gami AS, Hodge DO, Herges RM, et al. Obstructive sleep apnea, obesity, and the risk of incident atrial fibrillation. J Am Coll Cardiol 2007; 49:565-571.

14. Stevenson IH, Teichtahl H, Cunnington D, et al. Prevalence of sleep disordered breathing in paroxysmal and persistent atrial fibrillation patients with normal left ventricular function. Eur Heart J 2008; 29:1662-1669.

15. Kanagala R, Murali NS, Friedman PA, et al. Obstructive sleep apnea and the recurrence of atrial fibrillation. Circulation 2003; 107:2589-2594.

16. Gupta RM, Parvizi J, Hanssen AD, Gay PC. Postoperative complications in patients with obstructive sleep apnea syndrome undergoing hip or knee replacement: a case-control study. Mayo Clin Proc 2001; 76:897-905.

17. Chung F, Yegneswaran B, Liao P, et al. Validation of the Berlin questionnaire and American Society of Anesthesiologists checklist as screening tools for obstructive sleep apnea in surgical patients. Anesthesiology 2008; 108:822-830

18. Netzer NC, Stoohs RA, Netzer CM, Clark K, Strohl KP. Using the Berlin questionnaire to identify patients at risk for the sleep apnea syndrome. Ann Intern Med 1999; 131:485-491.

19. Gross JB, Bachenberg KL, Benumof JL, et al; American Society of Anesthesiologists Task Force on Perioperative Management. Practice guidelines for the perioperative management of patients with obstructive sleep apnea: a report by the American Society of Anesthesiologists Task Force on Perioperative Management of patients with obstructive sleep apnea. Anesthesiology 2006; 104:1081-1093.

20. Chung F, Yegneswaran B, Liao P, et al. STOP questionnaire: a tool to screen patients for obstructive sleep apnea. Anesthesiology 2008; 108:812-821.

21. Meoli AL, Rosen CL, Kristo D, et al. Upper airway management of the adult patient with obstructive sleep apnea in the perioperative period—avoiding complications. Clinical Practice Review Committee, American Academy of Sleep Medicine. Sleep 2003; 26:1060-1065.

Correspondence: Shirin Shafazand, MD, MS, Division of Pulmonary, Critical Care, and Sleep Medicine, University of Miami Miller School of Medicine, P.O. Box 016960, Miami, FL 331016960; sshafazand@med.miami.edu 